Finally, all studies report that overexpression of hpo induces apoptosis in developing Drosophila tissues. In explaining this, some of the groups suggest a direct effect, where ectopic hpo directly lowers DIAP1 protein levels by transcriptional or post-transcriptional mechanisms. However, it is now widely accepted that most, if not all, apoptosis in Drosophila requires reaper, hid and grim $^{16-18}$. Consistent with this view, the Halder lab shows that hid mediates hpoinduced apoptosis. As hpo transcription in Drosophila does not correlate with cell death, there is little evidence to support the hypothesis that hpo triggers apoptosis independently of reaper, hid and grim. It is more probable that the Hpo-Sav-Wts complex has a permissive role, determining the threshold of cell death in response to reaper, hid and grim.
Identifying mutations and genes affecting tissue growth is an extremely important first step in elucidating the underlying molecular mechanisms. The next question is how these genes are regulated to determine proper tissue size. Unfortunately, the expression patterns of hpo, sav and wts are not very informative, as they do not show any obvious correlation with patterns of cell division and cell death. Therefore, it seems that the Hpo-Sav-Wts complex is regulated through post-transcriptional mechanisms. Given the experimental accessibility of the Drosophila system and the intense efforts in the field, it should not be too long until the precise mechanism by which these genes restrict excessive growth during development and tumour suppression becomes clear.

1. Neufeld, T. P., de la Cruz, A. F., Johnston, L. A. \& Edgar, B. A. Cel/ 93, 1183-1193 (1998).
2. Johnston, L. A., Prober, D. A., Edgar, B. A., Eisenman, R. N. \& Gallant, P. Cell 98, 779-790 (1999).

3. Xu, T., Wang, W., Zhang, S., Stewart, R. A. \& Yu, W. Development 121, 1053-1063 (1995).

4. Tapon, N., Ito, N., Dickson, B. J., Treisman, J. E. \& Hariharan, I. K. Cell 105, 345-355 (2001).

5. Justice, R. W., Zilian, O., Woods, D. F., Noll, M. \& Bryant, P. J. Genes Dev. 9, 534-546 (1995).

6. Kango-Singh, M. et al. Development 129, 5719-5730 (2002).

7. Tapon, N. et al. Cell 110, 403-406 (2002).

8. Udan, R. S. et al. Nature Cell Biol. 5, 914-920 (2003).

9. Wu, S., Huang, J., Dong, J. \& Pan, D. Cell 114, 445-456 (2003).

10. Harvey, K. F., Pfleger, C. M. \& Hariharan, I. K. Cell 114, 457-467 (2003).

11. Pantalacci, S. et al. Nature Cell Biol. 5, 921-927 (2003).

12. Hay, B. A. et al. Cell 83, 1253-1262 (2003).

13. Taylor, L. K., Wang, H. C. \& Erikson, R. L. Proc. Natl Acad. Sci. USA 93, 10099-10104 (1996).

14. Cheung, W. L. et. al. Cel/ 113, 507-517 2003

15. Ryoo, H. D., Bergmann, A., Gonen, H., Ciechanover, A. \& Steller, H. Nature Cell Biol. 4, 432-438 (2002).

16. White, K. et al. Science 264, 677-683 (1994).

17. Grether, M. E., Abrams, J. M., Agapite, J., White, K. \& Steller, H. Genes Dev. 9, 1694-1708 (1995).

18. Chen, P., Nordstrom, W., Gish, B. \& Abrams, J. M. Genes Dev. 10, 1773-1782 (1996).

\section{From shoot to root}

Plant stem cells are present in the meristem of shoots, roots and later flowers; and developmental patterning of these tissues requires a fine balance between stem cell maintenance and the initiation of cell differentiation within this population. In shoots, this is kept in check by interactions between the CLAVATA (CLV) signalling pathway, which promotes stem cell differentiation, and factors that maintain stem cell identity. But it is less clear whether a common pathway is used in the meristems of other tissues. Hints that a similar pathway might regulate the stem cell population in the root meristem have recently been made (Dev. Genes Evol. 213, 371-381 (2003)) and, reporting in Current Biology (Curr. Biol. 13, 1435-1441 (2003)), Casamitjana-Martinez et al. have begun to unravel the molecular details of this CLV-like pathway.

In shoot meristems, CLV3 encodes a small secreted protein of the CLE family that binds to, and activates, the CLV1-CLV2 receptor complex. Downstream signalling from this complex then restricts the size of an 'organizing centre' that promotes stem cell identity in surrounding cells. Other members of the CLE family are expressed in roots. Casamitjana-Martinez et al. overexpressed one of these, CLE19, specifically in the root, and found that this leads to a progressive loss of root meristem cells (see figure).

Surprisingly, they found that the organizing centre was normal in the roots of these plants, which suggested that, in contrast to the CLV pathway in shoots, CLE proteins might affect the differentiating cells rather than the stem cell organizer itself.

Next, Casamitjana-Martinez et al. conducted a suppressor screen to ask what factors might act together with CLE19. From this they identified two genes, SOL1 and SOL2, that antagonize the CLE19 phenotype. So how might these factors interact with the CLV pathway? To get a handle on this, the authors cloned SOL1 and found that it encodes a putative $\mathrm{Zn}^{2+}$ carboxypeptidase that is expressed in both shoots and roots. This suggests that it is well placed to act in the CLV pathway; the authors favour a model in

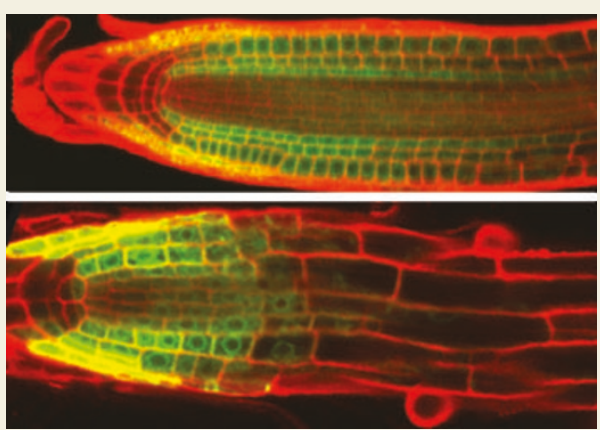

Overexpression of CLE19 in the root meristem reduces meristem size. Confocal images showing the root meristem boundary when green fluorescent protein (GFP) alone (left) or CLE19 and GFP (right) are expressed in the root meristem. Reproduced from Casamitjana-Martinez, E. et al. Curr. Biol. 13, 1435-1441 ( (2003) with permission from Elsevier Science.

which SOL1 is required to process inactive CLE protein into the active secreted form. This will now need to be tested directly, as will the function of SOL2 in the root.

Together, the results suggest that a CLV-like pathway is required for meristem maintenance in the root, as it is in the shoot. However, there are intriguing differences, the most striking of which is that, unlike in the shoot pathway, the action of CLE proteins seems to affect differentiating daughters of stem cells rather than the organizing centre or the stem cells themselves. No doubt further studies will show how divergent these pathways are, and they may also identify the root CLE receptor(s). This, together with further characterization of other CLE proteins that can affect root meristems, should reveal the true complexities of this crucial pathway.

ALISON SCHULDT 